\title{
Long non-coding RNA SUMO1P3 may promote cell proliferation, migration, and invasion of pancreatic cancer via EMT signaling pathway
}

\author{
CHUANG TIAN, YONG JIN and SONGSHAN SHI \\ Department of Urology, Fenjinting Hospital, Sihong, Jiangsu 223900, P.R. China \\ Received September 27, 2017; Accepted April 16, 2018
}

DOI: $10.3892 / 01.2018 .9378$

\begin{abstract}
Long non-coding RNAs (lncRNAs) have been suggested to serve important roles in the development of a number of human cancer types. An increasing amount of data has indicated that the lncRNA small ubiquitin-like modifier 1 (SUMO1) pseudogene 3 (SUMO1P3) has been involved in various types of human cancer. However, the function SUMO1P3 in the development of pancreatic cancer remains unclear. Firstly, reverse transcription-quantitative polymerase chain reaction was performed to determine the expression of SUMO1P3 in pancreatic cancer tissues and cell lines. Then, cell counting kit-8, wound-healing and transwell assays were conducted to explore the effect of SUMO1P3 on pancreatic cancer cell proliferation, migration and invasion. Finally, the EMT-associated proteins were evaluated by western blotting. The results of the present study revealed that SUMO1P3 expression was elevated in pancreatic tissues compared with the corresponding adjacent normal tissues. Additionally, the data indicated that the increased expression of SUMO1P3 is significantly associated with tumor progression and the poor survival of patients with pancreatic cancer. Furthermore, the present study identified that SUMO1P3 knockdown may suppress the proliferation, migration and invasion of pancreatic cancer cells. Additionally, downregulation of SUMO1P3 suppressed the epithelial-mesenchymal transition (EMT) and increased the expression of epithelial cadherin, and decreased the expression of neuronal cadherin, vimentin and $\beta$-catenin. Taken together, the results of the present study demonstrated that SUMO1P3 may participate in EMT and pancreatic cancer progression, thus suggesting that it may be a novel diagnostic and therapeutic biological target for pancreatic cancer.
\end{abstract}

Correspondence to: Dr Songshan Shi, Department of Urology, Fenjinting Hospital, 1 West Shuanggou Road, Sihong, Jiangsu 223900, P.R. China

E-mail: cancerther@21cn.com

Key words: small ubiquitin-like modifier 1 pseudogene 3, pancreatic cancer, prognosis, epithelial-mesenchymal

\section{Introduction}

Pancreatic cancer is one of the most common types of cancer that leads to increased mortality (1). Although significant improvements have been made in the diagnosis and treatment of pancreatic cancer in the last few decades, the prognosis of pancreatic cancer remains poor due to its aggressiveness and early systemic dissemination (2). The majority of patients with pancreatic cancer is diagnosed at an advanced stage of disease and is not eligible for curative resection, thus leading to high mortality rate (3). Therefore, it is imperative to investigate the molecular mechanisms underlying the development of pancreatic cancer in order to identify biological markers for the early diagnosis and the development of novel therapeutic agents in pancreatic cancer.

Long non-coding RNAs (lncRNAs) are a class of RNA molecules $>200$ nucleotides in length that do not encode proteins (4). Accumulating evidence has suggested that lncRNAs exhibit important roles in a number of fundamental cellular processes, including cell growth, apoptosis and differentiation by regulating gene expression at the transcriptional or post-transcriptional level $(5,6)$. Previous studies have demonstrated that several lncRNAs may be dysregulated in various types of human cancer $(7,8)$. Pseudogenes including small ubiquitin-like modifier 1 pseudogene (SUMO1P3) are considered as a separate class of lncRNAs (9).

SUMO1P3 was originally identified as a potential biomarker for the diagnosis of gastric cancer in 2013 (9). Furthermore, increased expression of SUMO1P3 has been reported in bladder cancer tissues, and it is significantly associated with a greater histological grade and advanced tumor-node metastasis (TNM) stage (10). An in vitro study further demonstrated that knockdown of SUMO1P3 inhibited bladder cancer cell proliferation and induced bladder cancer cell apoptosis (10). However, the biological function of SUMO1P3 in pancreatic cancer and its potential molecular mechanisms remain unclear.

In the present study, the expression of SUMO1P3 in pancreatic cancer tissues and the paired adjacent normal pancreatic tissues was evaluated, and its effects on epithelial-mesenchymal transition (EMT). The objective of the present study was to clarify the biological function and potential mechanism of SUMO1P3 in the development of pancreatic cancer. 


\section{Materials and methods}

Patient tissue samples. A total of 48 pairs of pancreatic cancer tissues and paired adjacent normal tissues were collected from patients undergoing surgical resection at The Pancreatic Cancer Center, Fenjinting Hospital between January 2009 and October 2013 (mean age 63.3 \pm 8.86 , age range, 37-71). All of the enrolled patients in the present study underwent surgery during which samples were collected. All specimens were frozen and stored in liquid nitrogen until further use. No patients received preoperative anticancer treatment, including chemotherapy or radiation prior to specimen collection. The present study was conducted with the approval of the Ethics and Research Committees of Fenjinting Hospital (Jiangsu, China) and was performed in accordance with the Declaration of Helsinki. All patients provided written informed consent prior to their participation in the present study. The clinical characteristics of all the patients are summarized in Table I.

Cell culture. The human pancreatic cancer cell lines BxPC-3, PANC-1, MiaPaCa-2, and ASPC-1 and the normal pancreatic ductal epithelial cell line HPDE6-C7 were purchased from the American Type Culture Collection (ATCC, Manassas, VA, USA). Cells were cultured in Dulbecco's modified Eagle's medium (DMEM; Gibco; Thermo Fisher Scientific, Inc., Waltham, MA, USA) supplemented with $10 \%$ fetal bovine serum (FBS; Gibco; Thermo Fisher Scientific, Inc.), $50 \mathrm{U} / \mathrm{ml}$ penicillin and $0.1 \mathrm{mg} / \mathrm{ml}$ streptomycin. Cells were cultured at $37^{\circ} \mathrm{C}$ in a humidified atmosphere containing $5 \% \mathrm{CO}_{2}$.

Small interfering (si)RNA transfection. siRNAs that targeted SUMO1P3 and a scrambled negative control were purchased from Shanghai GenePharma Co., Ltd. (Shanghai, China). The target sequence of siSUMO1P3 was 5'-TGGCCCTGATGT TCTAGCATGTGAT-3'. The RNAs were introduced into cells at a final concentration of $50 \mathrm{nM}$. Transfections were performed using the Lipofectamine 3000 kit (Invitrogen; Thermo Fisher Scientific, Inc., Waltham, MA, USA) according to the manufacturer's protocol. The knockdown efficiency was assessed by reverse transcription-quantitative polymerase chain reaction (RT-qPCR) $48 \mathrm{~h}$ after transfection.

$R N A$ extraction and RT-qPCR. Total RNA from tissues and cells was isolated using TRIzol reagent (Invitrogen; Thermo Fisher Scientific, Inc.), according to the manufacturer's protocol. Total RNA was reverse transcribed into cDNA using PrimeScript RT Reagent kit (Invitrogen; Thermo Fisher Scientific, Inc.), according to the manufacturer's protocol. qPCR was performed using SYBR Premix Ex Taq (Takara Biotechnology Co., Ltd., Dalian, China), according to the manufacturer's protocol. The primer sequences were as follows: SUMO1P3, 5'-ACTGGGAATGGAGGAAGA-3' (forward) and 5'-TGAGAAAGGATTGAGGGAAAAG-3' (reverse); GAPDH, 5'-CGCTCTCTGCTCCTCCTGTTC-3' (forward) and 5'-ATCCGTTGACTCCGACCTTCAC-3' (reverse). The thermocycling conditions were as follows: Initial denaturation at $95^{\circ} \mathrm{C}$ for $10 \mathrm{~min}$, followed by 40 cycles at $95^{\circ} \mathrm{C}$ for $15 \mathrm{sec}$ and extension at $60^{\circ} \mathrm{C}$ for $1 \mathrm{~min}$. Relative expression of PHGDH was normalized to the expression of GAPDH.
Relative expression of PHGDH was calculated using the $2^{-\Delta \Delta C q}$ method (11). All experiments were performed in triplicate. RT-qPCR was performed using the ABI PRISM 7500 PCR System (Applied Biosystems; Thermo Fisher Scientific, Inc.), according to the manufacturer's protocol. The median value was the cutoff between low and high SUMO1P3 expression in patients with pancreatic cancer. The median value was included in the low group.

Cell proliferation and colony formation assay. Following transfection with si-SUMO1P3 or si-negative control (NC), BxPC-3 panc-1 cells cell proliferation was accessed using Cell Counting Kit-8 (CCK-8; Dojindo Molecular Technologies, Inc., Kumamoto, Japan), according to the manufacturer's protocol. A total of 2,000 cells were plated into 96-well plates. Following culture for $24 \mathrm{~h}, 100 \mu \mathrm{l}$ CCK-8 was added into wells and the viable cells were evaluated at a wavelength of $450 \mathrm{~nm}$. For colony formation assay, 500 cells per plate were seeded in 6-well plates and incubated for two weeks. Then, colonies were fixed with $4 \%$ paraformaldehyde at room temperature for $15 \mathrm{~min}$ and stained with crystal violet. A total of five fields were randomly selected and cells were counted under a light microscope at low magnification (x100). Colonies that contained $>50$ cells were designated as survivors.

Cell migration and invasion assay. To measure the migratory ability of pancreatic cancer cells, a wound-healing assay was performed. BXPC-3 and PANC-1 cells were cultured in a 6-well plate until they reached $100 \%$ confluence. The monolayer cells were scratched using a $200 \mu \mathrm{l}$ sterile pipette tip to create the wound. Cells were cultured with DMEM without FBS at $37^{\circ} \mathrm{C}$ for $24 \mathrm{~h}$. The migration of cells across the gap wound was measured using light microscopy (x100 magnification). The invasive ability of pancreatic cancer cells was assessed using a Matrigel-coated Transwell chamber (BD Biosciences, San Jose, CA, USA). A total of $2 \times 10^{4}$ cells in $100 \mu \mathrm{l}$ of serum-free medium were plated in the upper chamber. DMEM medium supplemented with $10 \%$ FBS was plated in the lower chamber. Following incubation for $24 \mathrm{~h}$ at $37^{\circ} \mathrm{C}$ in a humidified atmosphere containing $5 \% \mathrm{CO}_{2}$. The non-invading cells on the upper surface of the well were scraped off with a cotton swab, and the invading cells on the lower surface were stained with $4 \%$ crystal violet at room temperature for $10 \mathrm{~min}$ and counted using a light microscope (x200 magnification). Each experiment was performed in triplicate.

Western blot analysis. Cell proteins were extracted with ice-cold lysis buffer (1 mM EDTA, pH 8.0, $50 \mathrm{mM}$ Tris/ $\mathrm{HCl}$, $\mathrm{pH} 7.4,150 \mathrm{mM} \mathrm{NaCl}, 1 \% \mathrm{NP}-40,0.1 \%$ SDS and $0.5 \%$ sodium deoxycholate, $\mathrm{pH}$ 7.4). After $30 \mathrm{~min}$ on ice, cell debris was removed by centrifugation at $15,000 \mathrm{x}$ g for $15 \mathrm{~min}$ at $4^{\circ} \mathrm{C}$. A basic protein quantification kit (BioVision, Inc., CA, USA) was used to determine total protein concentration. Equal amounts of the protein (20-40 $\mu \mathrm{g} / \mathrm{lane})$ were separated by SDS-PAGE (10\% gels) and transferred onto polyvinylidene difluoride (PVDF) membranes and the blots were blocked for $1 \mathrm{~h}$ using $5 \%$ fat-free milk at room temperature. The membranes were incubated with the following primary antibodies: Anti-vimentin (5741; Cell Signaling Technology, 

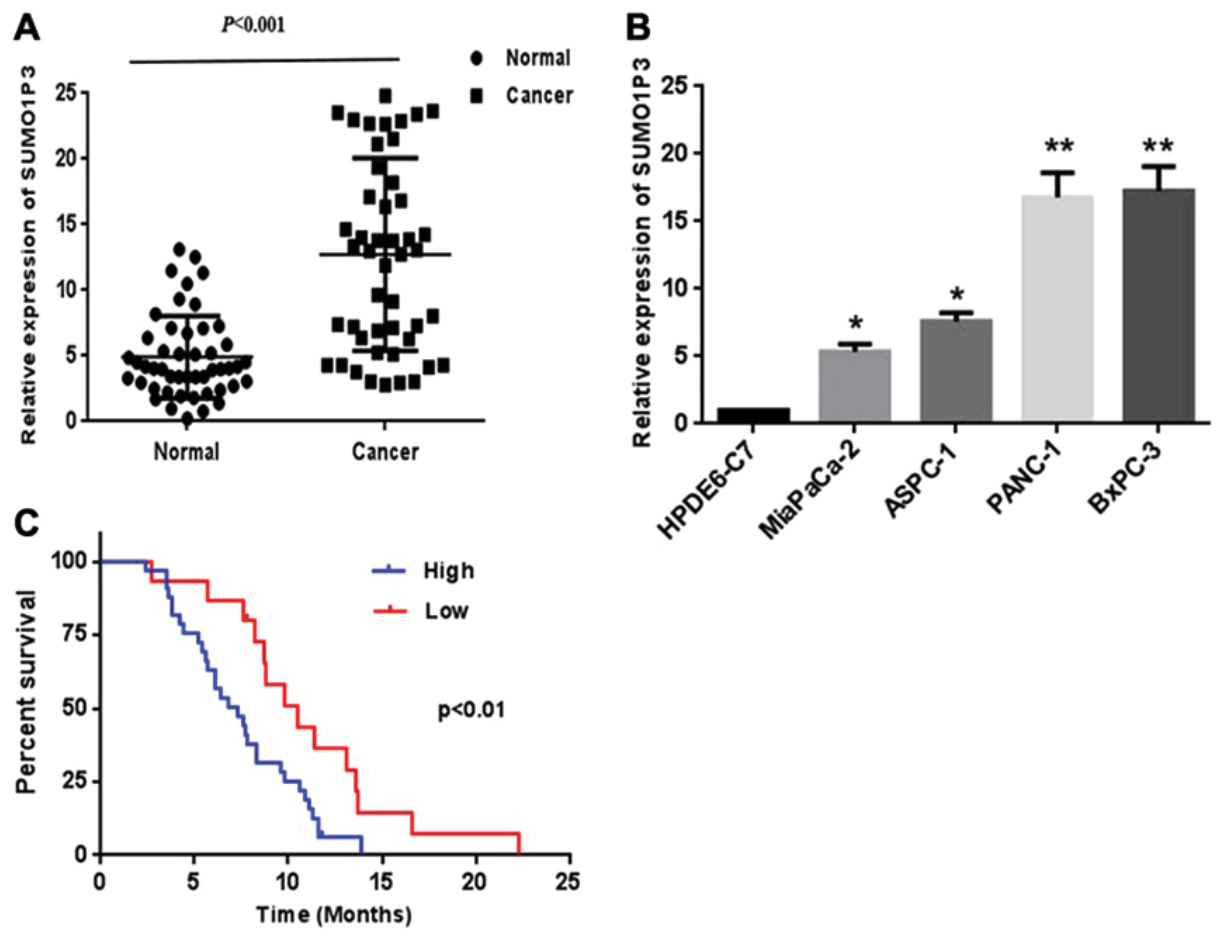

Figure 1. Relative expression of SUMO1P3 in pancreatic cancer tissues and cells. (A) Relative expression of SUMO1P3 in 48 pairs of pancreatic cancer tissues and adjacent non-tumor tissues by reverse transcription-quantitative polymerase chain reaction analysis. (B) SUMO103 is upregulated in pancreatic cancer cells compared with normal pancreatic ductal epithelial cell. (C) Kaplan-Meier curves for overall survival of two groups defined by low and high expression of SUMO1P3 in patients with pancreatic cancer. The median expression of SUMO1P3 is $12.34 \pm 8.76{ }^{*} \mathrm{P}<0.05,{ }^{* *} \mathrm{P}<0.01$. SUMO1P3, small ubiquitin-like modifier 1 pseudogene 3 .

Inc., Danvers, MA, USA; 1:1,000 dilution); Anti-neuronal (N)-cadherin (13116; Cell Signaling Technology; 1:1,000 dilution); Anti- $\beta$-catenin (8480; Cell Signaling Technology; 1:1,000 dilution); Anti-Epithelial (E)-cadherin (3195; Cell Signaling Technology; 1:1,000 dilution); and anti- $\beta$-actin antibody (sc-58673, Santa Cruz Biotechnology, Inc., Dallas, TX, USA) overnight at $4^{\circ} \mathrm{C}$. Following primary incubation, membranes were incubated with secondary antibodies (cat. no. AB6721; 1:3,000; Abcam) for $1 \mathrm{~h}$ at room temperature Finally, protein bands were developed with Amersham ECL Western Blotting Detection reagent (GE Healthcare Life Sciences, Little Chalfont, UK) and visualized using a gel imaging analysis system (Bio-Rad Laboratories, Inc., Hercules, CA, USA) and further analyzed using Image Lab software (version 3.0; Bio-Rad Laboratories, Inc.).

Statistical analysis. All results are shown as mean \pm standard deviation and were analyzed using GraphPad Prism version 6 (GraphPad Software, Inc., La Jolla, CA, USA) from at least three independent experiments. The $\chi^{2}$ test was performed to explore the associations between SUMO1P3 level and clinicopathological factors. The Kaplan-Meier method was used to calculate the survival curve, and log-rank test to determine statistical significance. Multivariate analysis was applied to determine the independent indicator for overall survival of patients with pancreatic cancer. The differences between groups were analyzed using one-way analysis of variance (ANOVA) followed by the Student-Newman-Keuls test. $\mathrm{P}<0.05$ was considered to indicate a statistically significant difference.

\section{Results}

Expression of SUMO1P3 is upregulated in pancreatic cancer tissues and cells. To explore the role of SUMO1P3 in pancreatic cancer, the relative expression level of SUMO1P3 in 48 pairs of pancreatic cancer tissues and adjacent non-tumor tissues was examined by RT-qPCR analysis. As presented in Fig. 1A, increased expression of SUMO1P3 was identified in pancreatic cancer tissues compared with corresponding adjacent non-tumor tissues. The expression of SUMO1P3 was assessed in 4 human pancreatic cancer cell lines and normal pancreatic ductal epithelial cell line HPDE6-C7. The results revealed that pancreatic cancer cell lines demonstrated a higher expression of SUMO1P3 compared with that in the normal pancreatic ductal epithelial cell (Fig. 1B).

Increased expression of SUMOIP3 is associated with the progression and poor prognosis of patients with pancreatic cancer. The present study further investigated the association between SUMO1P3 expression and clinicopathological factors in 48 patients with pancreatic cancer (Table I). The results revealed that the increased expression of SUMO1P3 was significantly associated with tumor size $(\mathrm{P}=0.019)$, lymph node metastasis $(\mathrm{P}=0.028)$ and TNM stage $(\mathrm{P}=0.034)$. However, there was no significant association between SUMO1P3 expression and age, sex, histological differentiation or tumor location. Kaplan-Meier survival curves (Fig. 1C) demonstrated that the survival of patients with pancreatic cancer with a lower expression of SUMO1P3 is significantly improved compared with that of the higher expression group (the median value 
Table I. Association between the expression of SUMO1P3 and the clinicopathological characteristics of patients with pancreatic cancer.

\begin{tabular}{|c|c|c|c|c|}
\hline \multirow[b]{2}{*}{ Clinicopathological parameters } & \multirow[b]{2}{*}{$\mathrm{n}$} & \multicolumn{2}{|c|}{ SUMO1P3 expression, $\mathrm{n}$} & \multirow[b]{2}{*}{ P-value } \\
\hline & & High & Low & \\
\hline & 48 & 33 & 15 & \\
\hline Age, years & & & & 0.633 \\
\hline$<60$ & 19 & 12 & 7 & \\
\hline$\geq 60$ & 29 & 21 & 8 & \\
\hline Sex & & & & 0.416 \\
\hline Female & 17 & 13 & 4 & \\
\hline Male & 31 & 20 & 11 & \\
\hline Tumor size, $\mathrm{cm}$ & & & & 0.019 \\
\hline$<4$ & 30 & 17 & 13 & \\
\hline$\geq 4$ & 18 & 16 & 2 & \\
\hline Histological differentiation & & & & 0.205 \\
\hline Well and moderate & 33 & 21 & 12 & \\
\hline Poor & 15 & 12 & 3 & \\
\hline Location & & & & 0.371 \\
\hline Head-neck & 27 & 19 & 8 & \\
\hline Body-tail & 21 & 14 & 7 & \\
\hline Lymph node metastasis & & & & 0.028 \\
\hline Negative & 35 & 22 & 13 & \\
\hline Positive & 13 & 11 & 2 & \\
\hline TNM stage & & & & 0.034 \\
\hline I-II & 41 & 26 & 15 & \\
\hline III & 7 & 7 & 0 & \\
\hline
\end{tabular}

TNM, tumor-node metastasis; SUMO1P3, small ubiquitin-like modifier 1 pseudogene 3.

was the cutoff between low and high SUMO1P3 expression). Furthermore, multivariate analysis indicated that increased expression of SUMO1P3 was an independent indicator for overall survival of patients with pancreatic cancer (Table II).

Knockdown of SUMO1P3 impairs proliferation of BXPC-3 and PANC-1 cells in vitro. In order to explore the potential biological function of SUMO1P3 in the development of pancreatic cancer, SUMO1P3 expression was silenced in BXPC-3 and PANC-1 cells. As presented in Fig. 2A, mRNA expression level of SUMO1P3 was significantly decreased in cells transfected with SUMO1P3 siRNA compared with the control group. Knockdown of SUMO1P3 significantly suppressed the proliferative ability of BXPC-3 and PANC-1 cells (Fig. 2B), as assessed using a CCK-8 assay. Furthermore, a colony formation assay was also used to evaluate the cell proliferation ability. Knockdown of SUMO1P3 significantly decreased the colony formation ability of BXPC-3 and PANC-1 cells (Fig. 2C).

Knockdown of SUMO1P3 inhibits cell migration and invasion. Wound healing and Transwell assays were performed in order to measure the migratory and invasive ability of pancreatic cancer BxPC-3 cells and PANC-1 cells.
Table II. Cox multivariate regression analysis of the association of prognostic factors in pancreatic cancer.

\begin{tabular}{|c|c|c|c|}
\hline \multirow[b]{2}{*}{ Factors } & \multirow[b]{2}{*}{ P-value } & \multicolumn{2}{|c|}{$95 \%$ CI } \\
\hline & & Lower & Upper \\
\hline SUMO1P3 expression, high/low & 0.027 & 0.371 & 0.814 \\
\hline Age, years & 0.092 & 0.315 & 1.224 \\
\hline Sex & 0.831 & 0.272 & 1.283 \\
\hline Tumor size, cm & 0.063 & 0.329 & 1.072 \\
\hline Histological differentiation & 0.744 & 0.295 & 1.316 \\
\hline Location & 0.083 & 0.255 & 1.097 \\
\hline Lymph node metastasis & 0.062 & 0.216 & 1.013 \\
\hline TNM stage & 0.012 & 0.217 & 0.711 \\
\hline
\end{tabular}

TNM, tumor-node metastasis; CI, confidence interval; SUMO1P3, small ubiquitin-like modifier 1 pseudogene.

Knockdown of SUMO1P3 suppressed the migration ability of BxPC-3 (Fig. 3A and B) and PANC-1 (Fig. 3C and D) cells. Knockdown of SUMO1P3 led a significant inhibition of the 

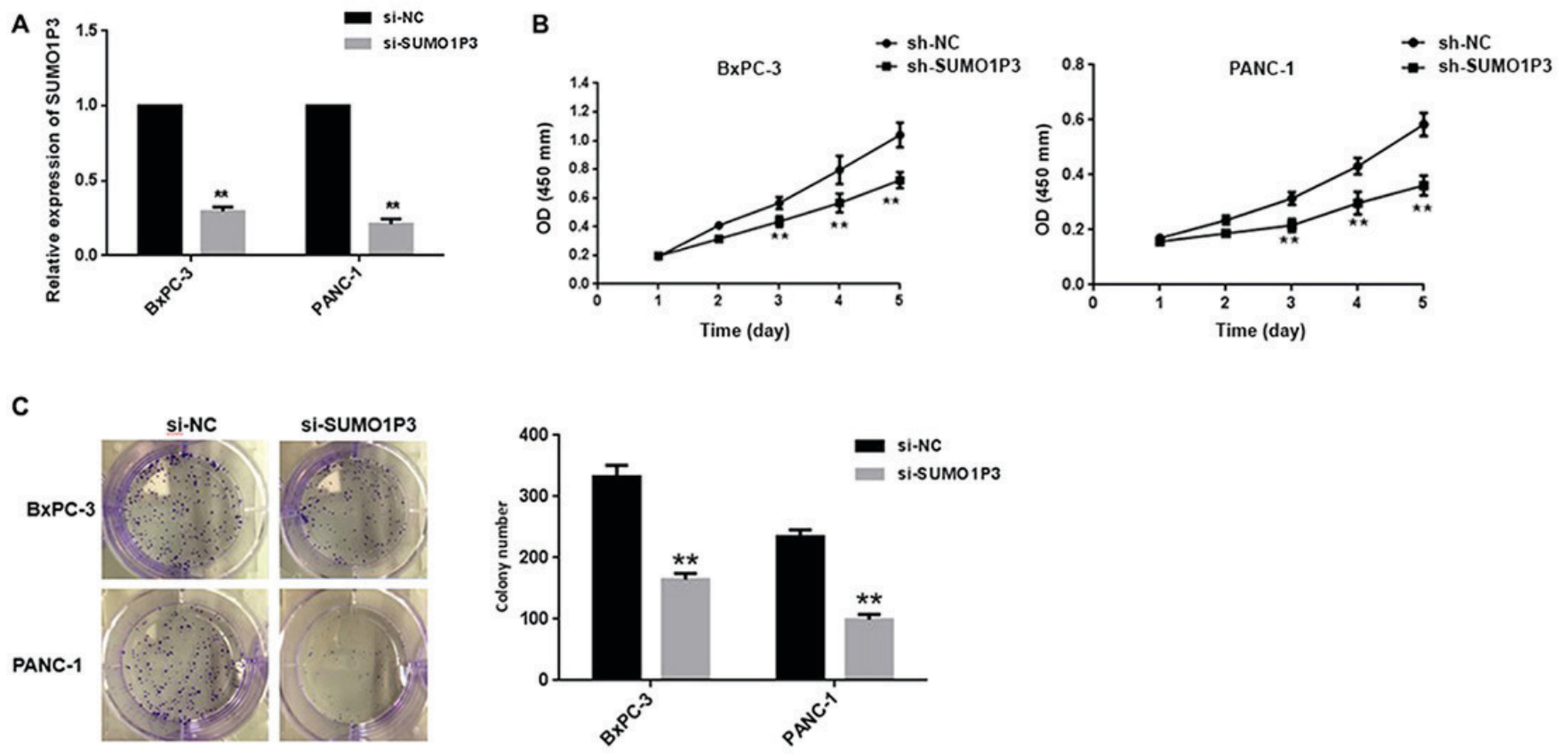

Figure 2. Effects of downregulation of SUMO1P3 on cell proliferation in pancreatic cancer. (A) The expression level of SUMO1P3 in BxPC-3 and PANC-1 cells was significantly decreased in response to si-SUMO1P3 compared with the si-NC group. (B) Knockdown of SUMO1P3 significantly suppressed cell proliferation of BxPC-3 and PANC-1 cells. (C) Colony-formation assay demonstrated that knockdown of SUMO1P3 significantly suppressed the colony-formation ability of BxPC-3 and PANC-1 cells compared with the negative control. ${ }^{* *} \mathrm{P}<0.01$. NC, negative control; si, small interfering; OD, optical density; SUMO1P3, small ubiquitin-like modifier 1 pseudogene 3.

A

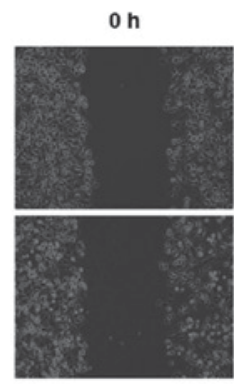

C

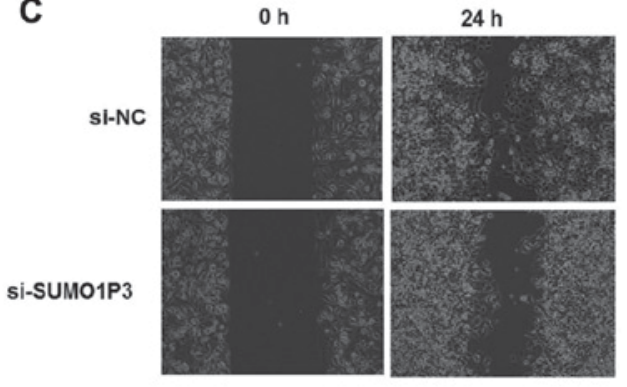

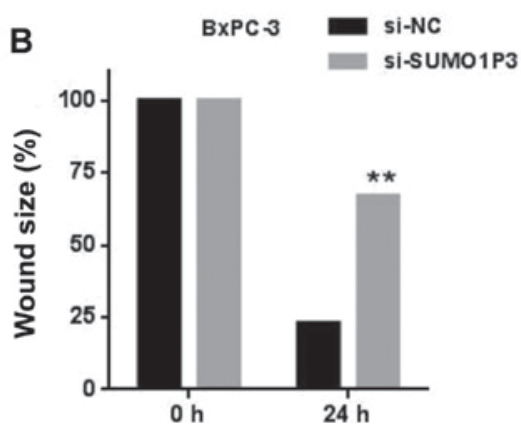

D

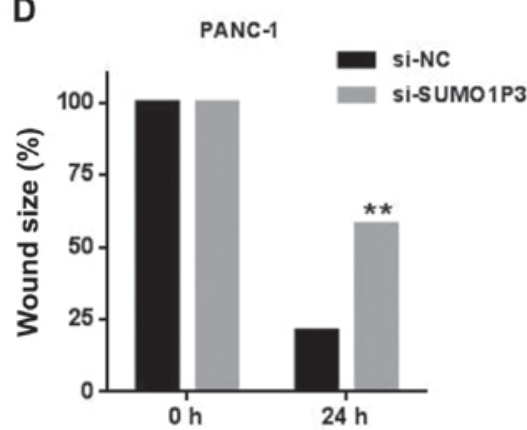

Figure 3. Effects of downregulation of SUMO1P3 on migration of pancreatic cancer cells. (A) Representative images and (B) quantification of wound healing in response to si-NC and si-SUMO1P3 in BxPC-3 cells. (C) Representative images and (D) quantification of wound healing in response to si-NC and si-SUMO1P3 in PANC-1 cells. (magnification, $\mathrm{x} 200$ ) ${ }^{* *} \mathrm{P}<0.01$. SUMO1P3, small ubiquitin-like modifier 1 pseudogene 3; NC, negative control; si, small interfering.

invasive ability of BxPC-3 and PANC-1 cells compared with that in the si-NC group (Fig. 4).

SUMOIP3 is associated with EMT. Since EMT is essential for tumor progression and metastasis (12), the effects of
SUMO1P3 on EMT were analyzed by western blotting. The results showed that downregulation of SUMO1P3 resulted in upregulation of epithelial markers (E-cadherin) and downregulation of mesenchymal markers (N-cadherin, vimentin and $\beta$-catenin) (Fig. 5). Taken together, these findings reveal 


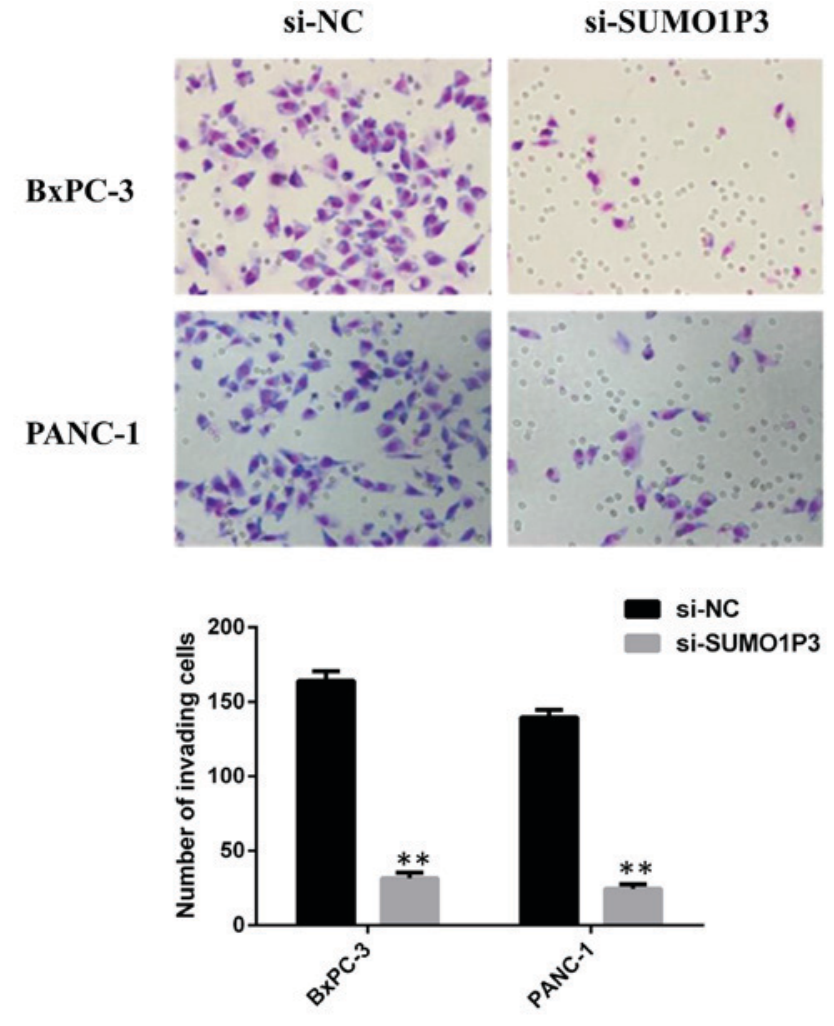

Figure 4. Effects of downregulation of SUMO1P3 on invasive ability of pancreatic cancer cells. Representative images and quantification of cell invasion in response to si-NC and si-SUMO1P3 in BxPC-3 and PANC-1 cells. ${ }^{* *} \mathrm{P}<0.01$ vs. si-NC. SUMO1P3, small ubiquitin-like modifier 1 pseudogene 3; NC, negative control; si, small interfering.

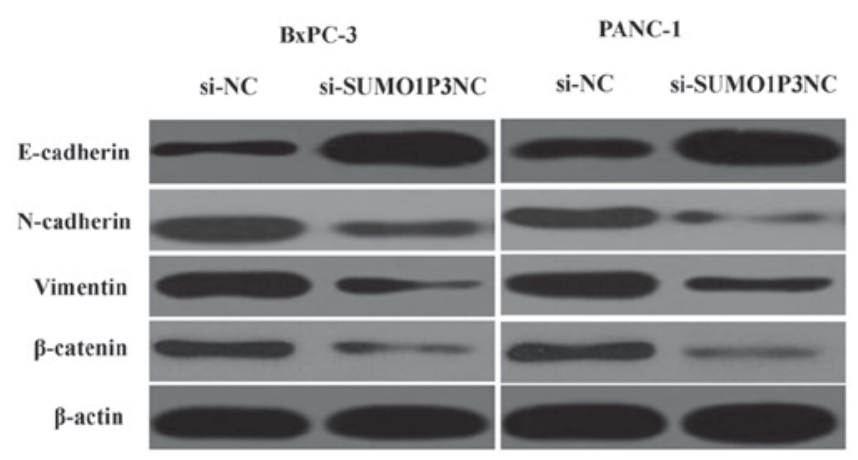

Figure 5. Effects of downregulation of SUMO1P3 on the expression of E-cadherin, $\mathrm{N}$-cadherin, vimentin and $\beta$-catenin. $\mathrm{E}$, epithelial; $\mathrm{N}$, neuronal; SUMO1P3, small ubiquitin-like modifier 1 pseudogene 3; NC, negative control; si, small interfering.

that SUMO1P3 may be a positive regulator of EMT, with an important biological function in the development of pancreatic cancer.

\section{Discussion}

Numerous studies have demonstrated that several lncRNAs serve a critical function in the development and progression of various types of cancer, including pancreatic cancer (13). For example, IncRNA-plasmacytoma variant translocation 1 functions as an endogenous sponge by competing with microRNA (miR)-488 to regulate Serpin Family E Member 1 mRNA Binding Protein 1 and therefore promote cell proliferation and migration in pancreatic cancer (14). LncRNA-taurine-upregulated gene 1 may enhance the cell proliferation and migration in pancreatic cancer by regulating EMT (15). Linc00673 may regulate non-small cell lung cancer proliferation, migration, invasion and EMT by functioning as an endogenous sponge by competing with miR-150-5p (16).

LncRNA SUMO1P3 has been previously reported to be upregulated and may serve as a potential therapeutic target in several types of cancer $(9,10)$. In the present study, SUMO1P3 was demonstrated to be upregulated in pancreatic cancer tissues compared with paired adjacent non-tumor tissue and increased expression of SUMO1P3 was significantly associated with tumor size, lymph node metastasis and TNM stage. Furthermore, the survival of patients with pancreatic cancer with a lower expression of SUMO1P3 was significantly improved compared with that of the higher expression group. Furthermore, knockdown of SUMO1P3 inhibited cell proliferation, migration and invasion. These results suggested that SUMO1P3 may serve an important role in the development of pancreatic cancer.

EMT is a vital pathological progress in the development and progression in numerous types of human cancer, including pancreatic cancer $(15,16)$. During this process, cancer cells lose cell polarity and cell-cell adhesion and acquire mesenchymal characteristics, including motility and invasiveness $(17,18)$. Cadherin switch (loss E-cadherin and gain of N-cadherin expression) represents an important characteristic in EMT (19). E-cadherin, a canonical epithelial marker, has been widely accepted as a critical suppressor of motility and invasiveness of epithelial cells in numerous types of cancer (20). Transcriptional downregulation or genomic deletion of E-cadherin expression may result in key pathological changes in tumor cells (21). However, $\mathrm{N}$-cadherin may promote invasion and distal metastasis in various types of human cancer (22). Vimentin and $\beta$-catenin are well-established markers for $\operatorname{EMT}(23,24)$.

Therefore, the present study further assessed whether the biological function of SUMO1P3 on pancreatic cancer cells was via EMT induction. The results demonstrated that downregulation of SUMO1P3 led to upregulation of epithelial markers (E-cadherin) and downregulation of mesenchymal markers ( $\mathrm{N}$-cadherin, vimentin and $\beta$-catenin). These results revealed that SUMO1P3 may function as an oncogene in pancreatic cancer by regulating EMT.

In conclusion, the expression of SUMO1P3 was increased in pancreatic cancer tissues and cell lines, and increased expression of SUMO1P3 was associated with the malignant status and poor prognosis in patients with pancreatic cancer. Knockdown of SUMO1P3 suppressed cell proliferation, migration and invasion in pancreatic cancer by regulating EMT in vitro.

\section{Acknowledgements}

Not applicable.

\section{Funding}

The present study was supported by The Fund for 333 Engineering Project in Jiangsu Province (grant no. BRA2017266). 


\section{Availability of data and materials}

The datasets used and/or analyzed during the present study are available from the corresponding author on request.

\section{Authors' contributions}

$\mathrm{CT}$ and YJ performed the majority of the research and were the major contributors in writing the manuscript. CT, YJ and SS prepared experimental materials and reviewed the article. CT and SS made substantial contributions to the design of the work, drafting the manuscript and revising it critically for important intellectual content. SS gave final approval of the version to be published.

\section{Ethics approval and consent to participate}

The present study was conducted with the approval of the Ethics and Research Committees of Fenjinting Hospital (Jiangsu, China) and was performed in accordance with the Declaration of Helsinki. All patients provided written informed consent prior to their participation in the present study.

\section{Patient consent for publication}

All patients provided written informed consent for the publication of their data.

\section{Competing interests}

The authors declare that they have no competing interests.

\section{References}

1. Siegel RL, Miller KD and Jemal A: Cancer statistics, 2016. CA Cancer J Clin 66: 7-30, 2016.

2. Xu W, Xu B, Yao Y, Yu X, Cao H, Zhang J, Liu J and Sheng H: Overexpression and biological function of IQGAP3 in human pancreatic cancer. Am J Transl Res 8: 5421-5432, 2016.

3. Yao W, Ji S, Qin Y, Yang J, Xu J, Zhang B, Xu W, Liu J, Shi S, Liu L, et al: Profilin-1 suppresses tumorigenicity in pancreatic cancer through regulation of the SIRT3-HIF1 $\alpha$ axis. Mol Cancer 13: 187, 2014.

4. Xiao H, Tang K, Liu P, Chen K, Hu J, Zeng J, Xiao W, Yu G, Yao W, Zhou H, et al: LncRNA MALAT1 functions as a competing endogenous RNA to regulate ZEB2 expression by sponging miR-200s in clear cell kidney carcinoma. Oncotarget 6 : 38005-38015, 2015.

5. Tsai MC, Manor O, Wan Y, Mosammaparast N, Wang JK, Lan F, Shi Y, Segal E and Chang HY: Long noncoding RNA as modular scaffold of histone modification complexes. Science 329: 689-693, 2010.

6. Fatica A and Bozzoni I: Long non-coding RNAs: New players in cell differentiation and development. Nat Rev Genet 15: 7-21, 2014.

7. Prensner JR and Chinnaiyan AM: The emergence of lncRNAs in cancer biology. Cancer Discov 1: 391-407, 2011.
8. Li CH and Chen Y: Targeting long non-coding RNAs in cancers: Progress and prospects. Int J Biochem Cell Biol 45: 1895-1910, 2013.

9. Mei D, Song H, Wang K, Lou Y, Sun W, Liu Z, Ding X and Guo J: Up-regulation of SUMO1 pseudogene 3 (SUMO1P3) in gastric cancer and its clinical association. Med Oncol 30: 709, 2013.

10. Zhan Y, Liu Y, Wang C, Lin J, Chen M, Chen X, Zhuang C, Liu L, $\mathrm{Xu} \mathrm{W}, \mathrm{Zhou} \mathrm{Q}$, et al: Increased expression of SUMO1P3 predicts poor prognosis and promotes tumor growth and metastasis in bladder cancer. Oncotarget 7: 16038-16048, 2016.

11. Lin C, Zhao GC, Xu YD, Wang DS, Jin DY, Ji Y, Lou WH and Wu WC: Increased expression of $\alpha$ Tubulin is associated with poor prognosis in patients with pancreatic cancer after surgical resection. Oncotarget 7: 60657-60664, 2016.

12. Livak KJ and Schmittgen TD: Analysis of relative gene expression data using real-time quantitative PCR and the 2(-Delta Delta C(T)) method. Methods 25: 402-408, 2001.

13. Hu X, Sood AK, Dang CV and Zhang L: The role of long noncoding RNAs in cancer: The dark matter matters. Curr Opin Genet Dev 48: 8-15, 2018.

14. Zhao L, Kong H, Sun H, Chen Z, Chen B and Zhou M: LncRNA-PVT1 promotes pancreatic cancer cells proliferation and migration through acting as a molecular sponge to regulate miR-448. J Cell Physiol 233: 4044-4055, 2018.

15. Qin CF and Zhao FL: Long non-coding RNA TUG1 can promote proliferation and migration of pancreatic cancer via EMT pathway. Eur Rev Med Pharmacol Sci 21: 2377-2384, 2017.

16. Lu W, Zhang H, Niu Y, Wu Y, Sun W, Li H, Kong J, Ding K, Shen HM, Wu H, et al: Long non-coding RNA linc00673 regulated non-small cell lung cancer proliferation, migration, invasion and epithelial mesenchymal transition by sponging miR-150-5p. Mol Cancer 16: 118, 2017.

17. Huang C, Xie D, Cui J, Li Q, Gao Y and Xie K: FOXM1c promotes pancreatic cancer epithelial-to-mesenchymal transition and metastasis via upregulation of expression of the urokinase plasminogen activator system. Clin Cancer Res 20: 1477-1488, 2014.

18. Ma R, Chen J, Jiang S, Lin S, Zhang X and Liang X: Up regulation of NAT10 promotes metastasis of hepatocellular carcinoma cells through epithelial-to-mesenchymal transition. Am J Transl Res 8: 4215-4223, 2016.

19. Tam WL and Weinberg RA: The epigenetics of epithelial-mesenchymal plasticity in cancer. Nat Med 19: 1438-1449, 2013.

20. Liu JF, Mao L, Bu LL, Ma SR, Huang CF, Zhang WF and Sun ZJ: $\mathrm{C} 4.4 \mathrm{~A}$ as a biomarker of head and neck squamous cell carcinoma and correlated with epithelial mesenchymal transition. Am J Cancer Res 5: 3505-3515, 2015.

21. Guo F, Parker Kerrigan BC, Yang D, Hu L, Shmulevich I, Sood AK, Xue F and Zhang W: Post-transcriptional regulatory network of epithelial-to-mesenchymal and mesenchymal-to-epithelial transitions. J Hematol Oncol 7: 19, 2014.

22. Shan ZZ, Yan XB, Yan LL, Tian Y, Meng QC, Qiu WW, Zhang $Z$ and Jin ZM: Overexpression of Tbx3 is correlated with Epithelial-Mesenchymal Transition phenotype and predicts poor prognosis of colorectal cancer. Am J Cancer Res 5: 344-353, 2014.

23. Dai C, Cao J, Zeng Y, Xu S, Jia X and Xu P: E-cadherin expression as a prognostic factor in patients with ovarian cancer: A meta-analysis. Oncotarget 8: 81052-81061, 2017.

24. Zhang X, Liu G, Kang Y, Dong Z, Qian Q and Ma X: N-cadherin expression is associated with acquisition of EMT phenotype and with enhanced invasion in erlotinib-resistant lung cancer cell lines. PLoS One 8: e57692, 2013. 